\title{
Hematopoietic stem cell gene therapy: selecting only the best
}

\author{
Arthur Bank \\ Departments of Medicine and of Genetics \& Development, Columbia University \\ College of Physicians and Surgeons, New York, New York, USA
}

\begin{abstract}
Hematopoietic stem cell (HSC) gene therapy can potentially cure a variety of human hematopoietic diseases, such as sickle cell disease. Selection and expansion of gene-corrected HSCs has now been accomplished for the first time using HSC from large animals - dogs and humans - with a novel drug-resistance gene, $M G M T$, which is not expressed in normal HSCs (see the related articles beginning on pages 1561 and 1581). Highly efficient lentiviral transfer and expression of MGMT into relatively few HSCs led to repopulation of most of the hematopoietic compartment with gene-corrected cells following suitable drug treatment. This selection system may be useful in human clinical trials to permit gene therapy in autologous and allogeneic bone marrow transplantation settings.
\end{abstract}

J. Clin. Invest. 112:1478-1480 (2003). doi:10.1172/JCI200320336.

Curing human diseases using gene transfer into human hematopoietic stem cells (HSCs) has long been a goal of scientists and physicians. HSCs remain among the most promising target cells for gene therapy because of their unique differentiation and expansion capabilities, which ensure that the transfer and expression of genes added to a small number of cells result in gene correction of much greater numbers of cells by self-renewal and differentiation. Targeting genes to cells more differentiated than HSCs does not result in long-term expression of the transferred genes, since these cells further differentiate and die rather than

Address correspondence to: Arthur Bank, Departments of Medicine and of Genetics \& Development, Columbia University College of Physicians and Surgeons, Armand Hammer Health Science Center, HHSC 16-1604, 701 West 168th Street, New York, New York 10032, USA.

Phone: (212) 305-4186; Fax: (212) 923-2090;

E-mail:ab13@columbia.edu.

Conflict of interest: The author has an equity interest in Genetix Pharmaceuticals Inc., which is active in the development of effective gene therapy technologies.

Nonstandard abbreviations used: hematopoietic stem cell (HSC); multidrug resistance 1 (MDR1); $O^{6}$-methylguanine-DNA methyltransferase (MGMT); nitrosourea (NU); $\mathrm{O}^{6}$-benzylguanine $\left(\mathrm{O}^{6} \mathrm{BG}\right)$; 1,3-bis-(2chloroethyl)-1-nitrosourea (BCNU). self-renew. In order to transduce HSCs it is necessary to use vectors that integrate into chromosomal DNA and are faithfully transferred to daughter cells. Currently, oncoretroviruses and lentiviruses are used as suitable vectors; adenoviruses and other transfer vectors are either carried in the cell as episomes or integrate poorly. HSCs are relatively rare populations and are often quiescent, which makes it difficult to transfer genes into these cells with high efficiency. Lentiviral vectors have an advantage over oncoretroviruses in that they target nondividing cells including quiescent HSC.

\section{Lentiviral gene transfer}

High-titer lentiviruses have been successful in curing mouse models of sickle cell disease and $\beta$-thalassemia $(1,2)$. Gene transfer into the HSCs of larger animals, including humans, has been much more problematic. Although human gene therapy trials have demonstrated successful transduction of human HSCs, the numbers of gene-corrected human HSCs observed following transplantation have been relatively low. To expand this population of gene-corrected cells, selectable marker genes have been used. The most successful clini- cal gene therapy trials to date used the human multidrug resistance 1 gene (MDR1), the P-glycoprotein product of which is required for the removal of certain drugs from cells $(3,4)$. P-glycoprotein expression permits the selection and expansion of the genecorrected HSCs and their progeny by drug treatment. In these trials, selection with anthracyclines and taxanes, drugs toxic to untransduced cells without significant endogenous MDR1 activity, led to a modest selection and expansion of the MDR1transduced human HSCs $(3,4)$.

\section{A potent HSC selection system}

More recently, a powerful selection system has been exploited to select for transduced HSCs using a mutant form of the enzyme $O^{6}$-methylguanine-DNA methyltransferase (MGMT), encoded by the MGMT gene, the expression of which is markedly reduced in normal HSCs. MGMT confers resistance to the cytotoxic effects of the anticancer drugs the nitrosoureas (NUs). In murine studies, transduction of very few HSCs with the mutant MGMT vector results in profound selection and expansion of gene-corrected cells following exposure to $\mathrm{NU}$ and $\mathrm{O}^{6}$-benzylguanine $\left(\mathrm{O}^{6} \mathrm{BG}\right)$ (5-8). In this issue of the JCI, two independent studies demonstrate for the first time the power of lentiviral vectors expressing the mutant MGMT gene in HSC selection and expansion in dogs and in human HSC $(9,10)$. In the study by Neff et al. (9), donor dog HSCs transduced with mutant MGMTexpressing vectors were strongly selected in vivo after transfer into normal recipients in an allogeneic bone marrow setting after treatment with 1,3 bis-(2-chloroethyl)-1-nitrosourea (BCNU) and $\mathrm{O}^{6} \mathrm{BG}$. In the second study, by Zielske et al. (10), human $\mathrm{CD} 4^{+}$cells (among which the majority of human HSCs are included) were transduced with the same mutant $M G M T$ vector, and selection and expansion of gene-corrected human HSCs was demonstrated using NOD/SCID mice to measure engraftment and expansion of human HSCs and their progeny (Figure 1). 


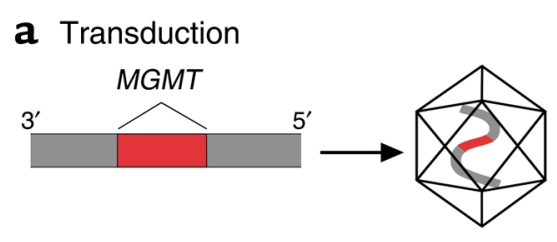

b Gene transfer into HSC

C In vivo selection and expansion
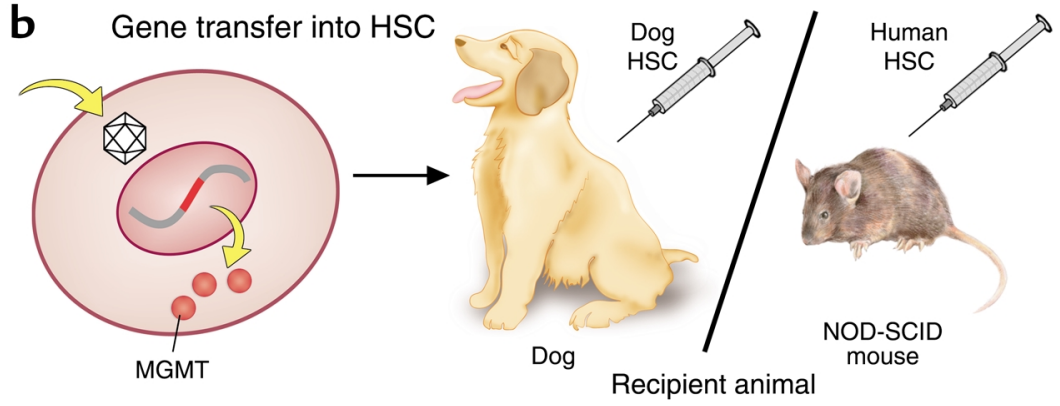

Before

After

Gene-corrected:

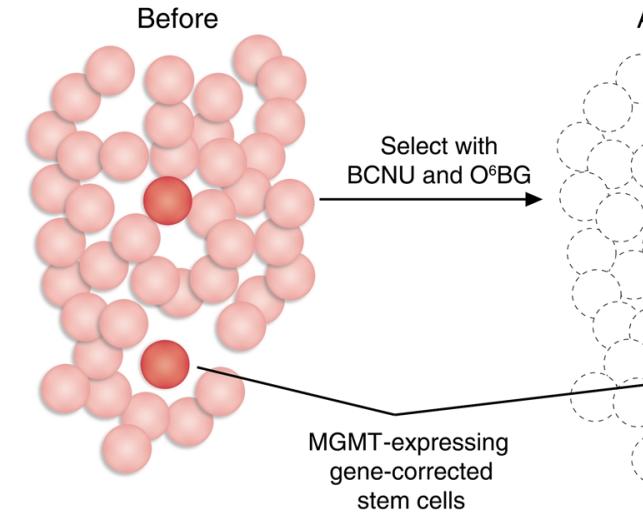

Red blood cells

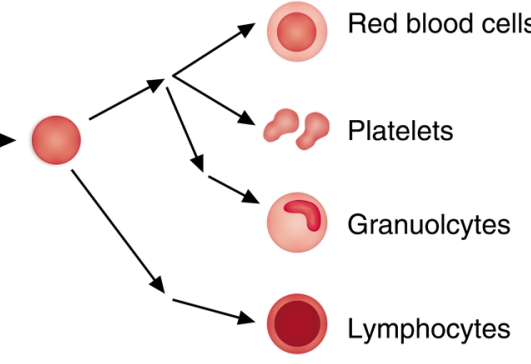

Figure 1

Gene therapy using HSCs. (a) Transduction of cells with lentiviral supernatants containing the transgene MGMT that integrates into chromosomal DNA. (b) Gene transfer into HSCs with subsequent injection of populations of hematopoietic cells containing gene-corrected HSCs into animals, either dogs or, for human HSCs, NOD/SCID mice. Cells given by vein home to the marrow. (c) In vivo selection. Before: Small numbers of MGMT-expressing gene-corrected cells. After: Almost complete killing of untransduced $\mathrm{HSCs}$ with $\mathrm{BCNU}$ and $\mathrm{O}^{6} \mathrm{BG}$ therapy. Self-renewal and expansion of MGMT gene-corrected HSCs repopulate all hematopoietic lineages.

The MGMT cell selection system is already being tested for protection of marrow from the toxic effects of NU treatment in a human phase 1 clinical trial in patients being treated with NUs (S. Gerson, unpublished observations). One future possibility is the use of this system to permit successful human allogeneic transplantation by MGMT gene transfer into umbilical cord blood that may have insufficient HSCs to fully engraft adults. Subsequent in vivo selection and expansion of the transduced donor HSCs would be achieved using $\mathrm{NU}$ and $\mathrm{O}^{6} \mathrm{BG}$ administration, analogous to the experiment performed in dogs and described in this issue by Neff et al. (9). Another potential application in humans would be the addition of the MGMT gene to retroviral or lentiviral vectors containing a nonselectable gene such as the human $\beta$-globin gene in an autotransplantation setting, followed by selection for the gene-corrected cells after transplantation.

\section{Gene therapy today}

Despite the recent development of leukemia in two of the nine subjects enrolled in a human clinical trial of retroviral gene therapy for X-linked SCID (11), the benefits afforded to almost all patients in this trial, in addition to the lack of significant toxicity reported in other similar trials, lend weight to the general opinion that human retroviral gene therapy trials using HSCs should continue in the hope of increasing therapeutic efficacy and safety $(12,13)$. Both reports in this issue of the JCI also show that relatively low numbers of integrations of the $M G M T$ gene result in sufficient expression of the enzyme to successfully select and expand the gene-corrected HSCs (9, 10). This is important because of the presumed potential for increased insertional mutagenesis with increased numbers of integrations. In the X-linked SCID gene therapy trial, both patients who developed leukemia demonstrated insertion of an unregulated $\gamma C$ receptor gene into the locus of a known prolifer- ative gene, LMO2. In both cases, there was a 2- to 3-year interval between the gene-correction event and the development of leukemia. These circumstances suggest that the leukemic clones were produced by the rare event of gene insertion at the LMO2 locus and were selected for proliferation over time. Methods that may be employed in future studies to reduce the type of insertional mutagenesis associated with these two individuals include the use of (a) insulators, which would prevent the upstream or downstream activation of the inserted gene(s); and (b) gene- and tissue-specific promoters, such as the human $\beta$-globin gene promoter in human $\beta$-globin gene therapy trials, as alternatives to more generally expressed promoters such as the retroviral longterminal repeats used in the X-linked SCID trial. These modifications and the use of HSC selection as described in the two papers in this issue of the JCI ( 9 , 10) may permit safe and effective HSC gene therapy of hematological disorders in the near future. 
1. May, C., et al. 2000. Therapeutic haemoglobin synthesis in beta-thalassaemic mice expressing lentivirus-encoded human beta-globin. Nature. 406:82-86.

2. Pawliuk, R., et al. 2001. Correction of sickle cell disease in transgenic mouse models by hematopoietic gene therapy. Science. 294:2368-2371.

3. Moscow, J.A., et al. 1999. Engraftment of MDR1 and NeoR gene-transduced hematopoietic cells after breast cancer chemotherapy. Blood. 94:52-61.

4. Abonour, R., et al. 2000. Efficient retrovirusmediated transfer of the multidrug resistance 1 gene into autologous human long-term repopulating hematopoietic stem cells. Nat. Med. 6:652-658.

5. Allay, J.A., Dumenco, L.L., Koc, O.N., Liu, L., and
Gerson, S.L. 1995. Retroviral transduction and expression of the human alkyltransferase cDNA provides nitrosourea resistance to hematopoietic cells. Blood. 85:3342-3351.

6. Allay, J.A., Davis, B.M., and Gerson, S.L. 1997. Human alkyltransferase-transduced murine myeloid progenitors are enriched in vivo by BCNU treatment of transplanted mice. Exp. Hematology. 25:1069-1076.

7. Gerson, S.L. 2002. Clinical relevance of MGMT in the treatment of cancer. J. Clin. Oncol. 20:2388-2399.

8. Persons, D.A., et al. 2003. Successful treatment of murine beta-thalassemia using in vivo selection of genetically modified, drug-resistant hematopoietic stem cells. Blood. 102:506-513.

9. Neff, T., et al. 2003. Methylguanine methyltransferase-mediated in vivo selection and chemoprotection of allogeneic stem cells in a large-animal model. J. Clin. Invest. 112:1581-1588. doi:10.1172/JCI200318782.

10. Zielske, S.P., et al. 2003. In vivo selection of MGMT(P140K) lentivirus-transduced human NOD/SCID repopulating cells without pretransplant irradiation conditioning. J. Clin. Invest 112:1561-1570. doi:10.1172/JCI200317922.

11. Kaiser, J. 2003. Gene therapy: seeking the cause of induced leukemias in X-SCID trial. Science. 299:495.

12. Friedmann, T. 2003. Gene therapy's new era: a balance of unequivocal benefit and unequivocal harm. Mol. Ther. 8:5-7.

13. Kohn, D.B., et al. 2003. American Society of Gene Therapy (ASGT) ad hoc subcommittee on retroviral-mediated gene transfer to hematopoietic stem cells. Mol. Ther. 8:180-187.

\title{
Therapeutic CD154 antibody for lupus: promise for the future?
}

\section{Garnett Kelsoe}

Department of Immunology, Duke University, Durham, North Carolina, USA

\begin{abstract}
Systemic lupus erythematosus (SLE) is a prototypical systemic autoimmune disease characterized by the production of pathogenic autoantibodies. A new study (see the related article beginning on page 1506) demonstrates that passive antibody specific for the TNF family member, CD154, ameliorates disease by reducing levels of self-reactive antibody in the serum. This study demonstrates a substantial potential for antiCD154 antibody in the treatment of humoral autoimmunity.
\end{abstract}

J. Clin. Invest. 112:1480-1482 (2003). doi:10.1172/JCI200320371.

The autoimmune disease systemic lupus erythematosus (SLE) is characterized by the production of highaffinity IgG antibodies against double-stranded DNA (dsDNA). Typically, these pathogenic autoantibodies are encoded by mutated I $g$ genes, and the frequency and pattern of mutations strongly suggest that the source of autoreactive antibodies is B cells that have participated in the germinal

Address correspondence to: Garnett Kelsoe, Department of Immunology, Duke University Medical Center 3010, Duke University, Durham, North Carolina 27710, USA. Phone: (919) 613-7815; Fax: (919) 613-7878; E-mail: ghkelsoe@duke.edu.

Conflict of interest: The author has declared that no conflict of interest exists.

Nonstandard abbreviations used: systemic lupus erythematosus (SLE); double-stranded DNA (dsDNA); germinal center (GC); follicular dendritic cell (FDC); dark zone (DZ); light zone (LZ); activation-induced cytidine deaminase (AID); somatic hypermutation (SHM); class switch recombination (CSR); SLE disease activity index (SLEDAI). center (GC) reaction. Indeed, GCs appear spontaneously in many strains of lupus-prone mice (1), and extraordinary numbers of GC B cells and their progeny are present in the blood of SLE patients (2).

GCs are organized collections of antigen-activated $\mathrm{T}$ and $\mathrm{B}$ lymphocytes in secondary lymphoid tissues (3) or sites of chronic inflammation $(4,5)$. Although transient GC-like reactions can be elicited by thymusindependent pathways (6-8), most $\mathrm{GC}$ responses are thymus-dependent. GC B cells require ongoing survival and proliferation signals that depend on CD154-CD40 signaling (9). CD154, a member of the TNF family, is inducibly expressed on the surface of CD4 T lymphocytes, whereas its ligand, CD40, is constitutively present on B lymphocytes (reviewed in ref. 10).

$\mathrm{T}$ cell-dependent GCs begin with the separate activation of $\mathrm{T}$ and $\mathrm{B}$ cells by antigen (11). In secondary lym- phoid tissues, binding of antigen modifies B cell chemotaxis, resulting in migration toward the $\mathrm{T}$ cell zone (12), where cognate T cell-B cell interaction expands both lymphocyte populations. Soon afterward, activated T and $\mathrm{B}$ cells emigrate from $\mathrm{T}$ cell areas into the reticula of follicular dendritic cells (FDCs) that define the B cell follicle (Figure 1). The immigrant $\mathrm{B}$ lymphocytes proliferate in the FDC reticulum to generate nascent GCs and, in the process, acquire distinctive phenotypes, including expression of the CD69 activation antigen and several differentiation markers, including CD38 and CD27. Remarkably, human $\mathrm{CD}^{3} 8^{+} \mathrm{GC}$ B cells can also express variable levels of CD154, especially under conditions of chronic stimulation $(13,14)$, and appear to have the capacity for continued self-activation.

GCs become polarized into histologic dark and light zones (DZ, LZ). The $\mathrm{DZ}$ is proximal to the $\mathrm{T}$ cell area and contains rapidly dividing $B$ cells called centroblasts that express little or no surface immunoglobulin. The more distal LZ contains the bulk of the activated FDC network, antigen-specific CD4 T cells, and nondividing B cells known as centrocytes. Centrocytes express surface immunoglobulin and are thought to be the progeny of $\mathrm{DZ}$ centroblasts. In turn, selected centrocytes likely reenter the DZ and regain the centroblast form.

The enzyme AID (activation-induced cytidine deaminase) drives both somatic hypermutation (SHM) and immunoglobulin class switch recombination (CSR) in GC B cells (15). SHM introduces point mutations and occasional small deletions into the 